\title{
On abstract generalized topological spaces generated by the density type operators
}

\author{
Jacek Hejduk (D), Anna Loranty*(D) \\ Faculty of Mathematics and Computer Science, Eódź University, Banacha 22, 90-238 Eódź, Poland
}

\begin{abstract}
In the paper we concentrate on a generalized topological space generated by a density type operator on a measurable space. The properties of such generalized topological space are investigated. Moreover, the properties of nowhere dense sets, meager sets and compact sets in this generalized topological space are studied.
\end{abstract}

Mathematics Subject Classification (2010). 54A05, 28A05, 54D10

Keywords. a generalized topological space, separation axioms, a nowhere dense set, the lower density operator

\section{Introduction}

Let $X$ be a non-empty set, $\mathcal{S}$ be an algebra of subsets of $X$ (i.e. the empty set belongs to $\mathcal{S}$ and $\mathcal{S}$ is closed under the finite unions of sets and the complements of sets) and $\mathcal{J} \subset \mathcal{S}$ be a ideal of subsets of $X$ (i.e. if $A \in \mathcal{J}$ and $B \subset A$ then $B \in \mathcal{J}$ and $\mathcal{J}$ is closed under the finite unions of sets). We will focus on a measurable space i.e. a triple $\langle X, \mathcal{S}, \mathcal{J}\rangle$, where $\mathcal{J} \subset \mathcal{S}$ is a proper ideal of sets such that all singletons belong to $\mathcal{J}$. Moreover, if it is necessary, we will assume that $\mathcal{J}$ is the $\sigma$-ideal of sets it means $\mathcal{J}$ is additionally closed under the countable unions of sets. The density type operators defined on some families of subsets of this space will also play a special role in our considerations.

The family of all subsets of a non-empty set $X$ will be denoted by $2^{X}$. For any $A, B \in 2^{X}$ the symbol $A \triangle B$ will stand for the set $(A \backslash B) \cup(B \backslash A)$. Moreover, for any measurable space $\langle X, \mathcal{S}, \mathcal{J}\rangle$ and $A, B \subset X$ we will write $A \sim B$ iff $A \triangle B \in \mathcal{J}$.

Let $\langle X, \mathcal{S}, \mathcal{J}\rangle$ be a measurable space. A measurable hull of a set $A \subset X$ is any set $B \in \mathcal{S}$ such that $A \subset B$ and for any $C \subset B \backslash A$ if $C \in \mathcal{S}$ then $C \in \mathcal{J}$. The set $B$ described above is called an $\mathcal{S}$-measurable hull of a set $A$. We will write it simply "a measurable hull of $A$ " when no confusion can arise. The family of all measurable hulls of a set $A \subset X$ will be denoted by $\mathcal{H}(A)$. We shall say that $\langle X, \mathcal{S}, \mathcal{J}\rangle$ has the hull property if $\mathcal{H}(A) \neq \emptyset$ for any set $A \subset X$.

In the next part of the paper a notion of a generalized topological space, introduced in [1] by Á. Császár, will be used. We shall say that a family $\gamma \subset 2^{X}$ is a generalized topology on $X$ if $\emptyset \in \gamma$ and $\bigcup_{t \in T} G_{t} \in \gamma$ whenever $\left\{G_{t}: t \in T\right\} \subset \gamma$. The pair $(X, \gamma)$ is

\footnotetext{
*Corresponding Author.

Email addresses: jacek.hejduk@wmii.uni.lodz.pl (J. Hejduk), anna.loranty@wmii.uni.lodz.pl (A. Loranty) Received: 19.06.2019; Accepted: 19.02.2020
} 
called a generalized topological space. If $X \in \gamma$ then we shall say that $(X, \gamma)$ is a strong generalized topological space.

In the theory of generalized topological spaces almost all notions (e.g. an interior of a set, a closure of a set, a boundary of a set, a compact set) are defined as in standard topological spaces (see $[1,2]$ ). The interior, the closure and the boundary of $A \subset X$ will be denoted by $\operatorname{int}_{\gamma}(A), \operatorname{cl}_{\gamma}(A)$ and $\operatorname{Fr}_{\gamma}(A)$, respectively. Moreover, we will write $\gamma$-open, $\gamma$-closed, etc. if we want to emphasize that the considerations concern the space $(X, \gamma)$. Similarly to the classical topological space we define a base of a generalized topological space or a connected set in such space (see $[3,8]$ ). Separation axioms for a generalized topological space are defined as in the case of the classical topological space [4]. Moreover, the definitions of a separable space, Lindelöf space, first countable and second countable space can be adopt from the classical topological space.

In the case of a topological space, the notion of a nowhere dense set may be introduced by different equivalent definitions. One can say that $A$ is a nowhere dense set if the interior of the closure of $A$ is an empty set. On the other hand one can say that $A$ is a nowhere dense set if any nonempty open set contains a nonempty open subset which is disjoint with $A$. In the case of a generalized topological space, these two conditions can lead to different notions. In [9] one can find two notions connected with nowhere density in generalized topological space. We say that a set $A \subset X$ is $\gamma$-nowhere dense if $\operatorname{int}_{\gamma}\left(\operatorname{cl}_{\gamma}(A)\right)=\emptyset$. A set $A \subset X$ is $\gamma$-strongly nowhere dense if for $V \in \gamma \backslash\{\emptyset\}$ there exists $U \in \gamma \backslash\{\emptyset\}$ such that $U \subset V$ and $A \cap U=\emptyset$. It is easy to see that if $A$ is $\gamma$-strongly nowhere dense then it is $\gamma$-nowhere dense. The converse theorem is not true in general (see [9]).

At the beginning of this section we mentioned that the particular operators will play a special role in our consideration, so we start with their definitions. First we shortly recall the definition of the lower density operator which is investigated by many mathematicians (e.g. $[5,12])$.

Definition 1.1. We shall say that an operator $\Phi: \mathcal{S} \rightarrow \mathcal{S}$ is the lower density operator on $\langle X, \mathcal{S}, \mathcal{J}\rangle$ if

$$
\begin{aligned}
& 1^{\circ} \Phi(\emptyset)=\emptyset \text { and } \Phi(X)=X ; \\
& 2^{\circ} \underset{A \in \mathcal{S}}{\forall} \quad \forall \in \mathcal{S}(A \cap B)=\Phi(A) \cap \Phi(B) ; \\
& 3^{\circ} \underset{\forall \in \mathcal{B}}{\forall} A \triangle B \in \mathcal{J} D \Phi(A)=\Phi(B) ; \\
& 4^{\circ} \underset{A \in \mathcal{S}}{\forall} \Phi(A) \triangle A \in \mathcal{J} .
\end{aligned}
$$

Obviously, the classical density operator defined in [12] is an example of the lower density operator. If $\langle X, \mathcal{S}, \mathcal{J}\rangle$ is a measurable space with the hull property and $\Phi$ is the lower density operator on $\langle X, \mathcal{S}, \mathcal{J}\rangle$, then the following theorem is true (see [11], p. 213).

Theorem 1.2. The family $\mathcal{T}_{\Phi}=\{A \in \mathcal{S}: A \subset \Phi(A)\}$ is a topology on $X$, which is called a topology generated by $\Phi$.

Proof. One can find the proof of this theorem in [11], but for the convenience of the reader we will present that any union of elements of $\mathcal{T}_{\Phi}$ belongs to $\mathcal{T}_{\Phi}$. Let $\left\{A_{w}\right\}_{w \in W} \subset \mathcal{T}_{\Phi}$. Since $\langle X, \mathcal{S}, \mathcal{J}\rangle$ is a measurable space with the hull property, we obtain that there exists a set $B$ being a measurable kernel of the set $\bigcup_{w \in W} A_{w}$ (i.e. $B \in \mathcal{S}, B \subset \bigcup_{w \in W} A_{w}$ and for any measurable set $C \subset \bigcup_{w \in W} A_{w} \backslash B$ we have that $\left.C \in \mathcal{J}\right)$. Obviously $\left(A_{w} \cap B\right) \triangle A_{w} \in \mathcal{J}$ for any $w \in W$ and

$$
B \subset \bigcup_{w \in W} A_{w} \subset \bigcup_{w \in W} \Phi\left(A_{w}\right)=\bigcup_{w \in W} \Phi\left(A_{w} \cap B\right) \subset \Phi(B) .
$$


Condition $4^{\circ}$ from Definition 1.1 implies that $\Phi(B) \backslash B \in \mathcal{J}$ and, in consequence, $\bigcup_{w \in W} A_{w} \in$ $\mathcal{S}$. Now, it is easy to see that

$$
\bigcup_{w \in W} \Phi\left(A_{w}\right) \subset \Phi\left(\bigcup_{w \in W} \Phi\left(A_{w}\right)\right)
$$

so $\bigcup_{w \in W} A_{w} \in \mathcal{T}_{\Phi}$

Obviously topology described in the above theorem is an example of an abstract density topology. The papers $[6,7,10,13]$ contain many results and properties relevant to abstract density topologies and lower density operators. Now, we are following the lower density operator $\Phi$ on $\langle X, \mathcal{S}, \mathcal{J}\rangle$. From now on, we will assume that $\langle X, \mathcal{S}, \mathcal{J}\rangle$ has the hull property.

Let $\Phi$ be the lower density operator on $\langle X, \mathcal{S}, \mathcal{J}\rangle$. Let us consider an operator $\Phi^{*}: 2^{X} \rightarrow$ $\mathcal{S}$ defined in the following way:

$$
\underset{A \subset X}{\forall} \Phi^{*}(A)=\Phi(B)
$$

where $B$ is an $\mathcal{S}$-measurable hull of a set $A$. By condition $3^{\circ}$ of Definition 1.1 we have that $\Phi^{*}$ is defined correctly. Clearly, if $A \in \mathcal{S}$ then $\Phi^{*}(A)=\Phi(A)$. Moreover, we have the following propositions.

Proposition 1.3. $\quad 1^{\circ} \Phi^{*}(\emptyset)=\emptyset$ and $\Phi^{*}(X)=X$;

$$
\begin{aligned}
& 2^{\circ} \underset{A \subset X}{\forall} \quad \underset{B \in \mathcal{S}}{\forall} \Phi^{*}(A \cap B)=\Phi^{*}(A) \cap \Phi(B) ; \\
& 3^{\circ} \underset{\forall \subset X \quad B \subset X}{\forall} A \triangle B \in \mathcal{J} \Rightarrow \Phi^{*}(A)=\Phi^{*}(B) ; \\
& 4^{\circ} \underset{A \subset X}{\forall} A \backslash \Phi^{*}(A) \in \mathcal{J} .
\end{aligned}
$$

Proof. Condition $1^{\circ}$ is obvious. Let $A \subset X$ and $B \in \mathcal{S}$. If $C \in \mathcal{S}$ is a measurable hull of $A$ then $C \cap B$ is a measurable hull of $A \cap B$. Hence $\Phi^{*}(A \cap B)=\Phi(C \cap B)=\Phi(C) \cap \Phi(B)=$ $\Phi^{*}(A) \cap \Phi(B)$, so condition $2^{\circ}$ is satisfied. To prove $3^{\circ}$ let us observe that if $A \triangle B \in \mathcal{J}$ and $C_{1}, C_{2}$ are measurable hulls of $A$ and $B$, respectively, then $C_{1} \triangle C_{2} \in \mathcal{J}$. It implies that $\Phi\left(C_{1}\right)=\Phi\left(C_{2}\right)$ and finally, $\Phi^{*}(A)=\Phi^{*}(B)$. In the case of $4^{\circ}$ if $C$ is an $\mathcal{S}$-measurable hull of $A$ then $A \backslash \Phi^{*}(A) \subset C \backslash \Phi(C) \in \mathcal{J}$.

Proposition 1.4. For every $A \subset X$ the following properties hold:

i) $\Phi\left(\Phi^{*}(A)\right)=\Phi^{*}(A)$;

ii) $\Phi^{*}\left(A \cap \Phi^{*}(A)\right)=\Phi^{*}(A)$.

Proof. Let $B$ be a measurable hull of $A$. Then $\Phi\left(\Phi^{*}(A)\right)=\Phi(\Phi(B))=\Phi(B)=\Phi^{*}(A)$. It means that i) is satisfied. In the case of ii) we have $\Phi^{*}\left(A \cap \Phi^{*}(A)\right)=\Phi^{*}(A \cap \Phi(B))=$ $\Phi^{*}(A) \cap \Phi(B)=\Phi^{*}(A) \cap \Phi^{*}(A)=\Phi^{*}(A)$.

Proposition 1.5. For every $A \subset X$ we have

(i) $A \cap \Phi^{*}(A)=\emptyset$ iff $A \in \mathcal{J}$;

(ii) $A \cap \Phi^{*}(A) \in \mathcal{S}$ iff $A \in \mathcal{S}$.

Proof. If $A \in \mathcal{J}$ then by Definition 1.1 we have $\Phi(A)=\emptyset$, so that $A \cap \Phi(A)=\emptyset$. Let $A \notin \mathcal{J}$. Then $A=\left(A \cap \Phi^{*}(A)\right) \cup\left(A \backslash \Phi^{*}(A)\right)$. Since, by Proposition 1.3, $A \backslash \Phi^{*}(A) \in \mathcal{J}$ we get that $A \cap \Phi^{*}(A) \notin \mathcal{J}$. It implies that $A \cap \Phi^{*}(A) \neq \emptyset$ and condition (i) is satisfied.

Now, we prove condition (ii). If $A \in \mathcal{S}$ then $\Phi^{*}(A)=\Phi(A)$ and, by Definition 1.1, we get that $\Phi(A) \in \mathcal{S}$. It implies that $A \cap \Phi^{*}(A) \in \mathcal{S}$. If $A \cap \Phi^{*}(A) \in \mathcal{S}$ then, by Proposition $1.3, A \backslash \Phi^{*}(A) \in \mathcal{J}$ and we get that $A \in \mathcal{S}$. 


\section{A generalized topological space connected with $\Phi^{*}$}

In this section, we will study the family

$$
\mathcal{T}_{\Phi^{*}}=\left\{A \subset X: A \subset \Phi^{*}(A)\right\}
$$

generated by the operator $\Phi^{*}$.

Remark 2.1. The family $\mathcal{T}_{\Phi^{*}}$ has not to be closed with respect to the finite intersection.

Indeed, let $\mathbb{R}$ be the set of all real numbers, $\mathcal{L}$ be the $\sigma$-algebra of Lebesgue measurable sets and $\mathbb{L}$ be the $\sigma$-ideal of Lebesgue measure zero sets in $\mathbb{R}$. If $\Phi$ is the density operator on $\langle\mathbb{R}, \mathcal{L}, \mathbb{L}\rangle$ and $B$ is a Bernstein set then $\Phi^{*}(B)=\Phi(\mathbb{R})=\mathbb{R}$ and $\Phi^{*}(\mathbb{R} \backslash B)=\Phi(\mathbb{R})=\mathbb{R}$. Additionally, for every $x \in \mathbb{R}$ we get that $B \cup\{x\} \in \mathcal{T}_{\Phi^{*}}$ and $(\mathbb{R} \backslash B) \cup\{x\} \in \mathcal{T}_{\Phi^{*}}$, but $(B \cup\{x\}) \cap((\mathbb{R} \backslash B) \cup\{x\})=\{x\} \notin \mathcal{T}_{\Phi^{*}}$.

However, it is easy to prove the following theorem:

Theorem 2.2. The family $\mathcal{T}_{\Phi^{*}}$ is a strong generalized topology on $X$ and $\mathcal{T}_{\Phi} \subset \mathcal{T}_{\Phi^{*}}$.

If we consider the classical density operator $\Phi$ on $\langle\mathbb{R}, \mathcal{L}, \mathbb{L}\rangle$, then it is easy to see that a Bernstein set belongs to $\mathcal{T}_{\Phi^{*}} \backslash \mathcal{T}_{\Phi}$. Proposition 1.3 implies that

Remark 2.3. If $W \in \mathcal{T}_{\Phi^{*}}$ and $A \in \mathcal{J}$ then $W \backslash A \in \mathcal{T}_{\Phi^{*}}$. Moreover, if $W \in \mathcal{T}_{\Phi^{*}} \backslash\{\emptyset\}$ then $W \notin \partial$.

Proof. Let $W \in \mathcal{T}_{\Phi^{*}}, A \in \mathcal{J}$ and $V=W \backslash A$. Clearly, $V \triangle W \in \mathcal{J}$, so Condition $3^{\circ}$ in Proposition 1.3 gives that $\Phi^{*}(V)=\Phi^{*}(W)$. Obviously, we have that $W \subset \Phi^{*}(W)$, because $W \in \mathcal{T}_{\Phi^{*}}$. Thus $V \subset W \subset \Phi^{*}(W)=\Phi^{*}(V)$, which gives that $V \in \mathcal{T}_{\Phi^{*}}$. Let now $W \in \mathcal{T}_{\Phi^{*}} \backslash\{\emptyset\}$. Suppose, contrary to our claim that $W \in \mathcal{J}$. Proposition 1.3 Conditions $1^{\circ}$ and $3^{\circ}$ imply that $\Phi^{*}(W)=\Phi^{*}(\emptyset)=\emptyset$. Since $W \subset \Phi^{*}(W)$ we obtain that $W=\emptyset$, which is impossible.

By Proposition 1.4 we have that

Remark 2.4. For every $A \subset X$ the sets $\Phi^{*}(A)$ and $A \cap \Phi^{*}(A)$ are the members of $\mathcal{T}_{\Phi^{*}}$.

Moreover, we have the following properties:

Proposition 2.5. For every $A \subset X$ we have

$$
\operatorname{int}_{\mathcal{T}_{\Phi^{*}}}(A)=A \cap \Phi^{*}(A) .
$$

Proof. By Remark 2.4 we have $A \cap \Phi^{*}(A) \in \mathcal{T}_{\Phi^{*}}$, so that $A \cap \Phi^{*}(A) \subset \operatorname{int}_{\mathcal{T}_{\Phi^{*}}}(A)$. Let $V \in \mathcal{T}_{\Phi^{*}}$ and $V \subset A$. Then $\Phi^{*}(V) \subset \Phi^{*}(A)$. So that $V \subset A \cap \Phi^{*}(A)$. Finally, $\operatorname{int}_{\mathcal{T}_{\Phi^{*}}}(A) \subset$ $A \cap \Phi^{*}(A)$.

Proposition 2.6. $\operatorname{Fr}_{\mathcal{T}_{\Phi^{*}}}(A) \in \mathcal{J}$ for every set $A \subset X$.

Proof. Obviously, $\operatorname{Fr}_{\mathcal{T}_{\Phi^{*}}}(A)=\operatorname{cl}_{\mathcal{T}_{\Phi^{*}}}(A) \backslash \operatorname{int}_{\mathcal{T}_{\Phi^{*}}}(A)=\left[A \cup\left(X \backslash \Phi^{*}(X \backslash A)\right] \backslash\left(A \cap \Phi^{*}(A)\right)=\right.$ $\left(A \backslash \Phi^{*}(A)\right) \cup\left[\left(X \backslash \Phi^{*}(X \backslash A)\right) \cap\left((X \backslash A) \cup\left(X \backslash \Phi^{*}(A)\right)\right] \subset\left(A \backslash \Phi^{*}(A)\right) \cup\left((X \backslash A) \backslash \Phi^{*}(X \backslash A)\right) \in\right.$ J.

The next property is the characterization of nowhere dense sets in the generalized topological space $\left\langle X, \mathcal{T}_{\Phi^{*}}\right\rangle$.

Theorem 2.7. Let $A \subset X$. Then the following conditions are equivalent:
i) $\underset{W \in \mathcal{T}_{\Phi^{*}}^{\forall} \backslash\{\emptyset\}}{\forall} \stackrel{\exists \in \mathcal{T}_{\Phi^{*}} \backslash\{\emptyset\}}{\exists}(V \subset W \wedge V \cap A=\emptyset)$;
ii) $\operatorname{int}_{\mathcal{T}_{\Phi^{*}}}\left(\operatorname{cl}_{\mathcal{T}_{\Phi^{*}}}(A)\right)=\emptyset$;
iii) $A \in \mathcal{J}$. 
Proof. i) $\Rightarrow$ ii). Let us suppose that $\operatorname{int}_{\mathcal{T}_{\Phi^{*}}}\left(\operatorname{cl}_{\mathcal{T}_{\Phi^{*}}}(A)\right) \neq \emptyset$. Hence there exists $W \in$ $\mathcal{T}_{\Phi^{*}} \backslash\{\emptyset\}$ such that $W \subset \operatorname{int}_{\mathcal{T}_{\Phi^{*}}}\left(\operatorname{cl}_{\mathcal{T}_{\Phi^{*}}}(A)\right)$. By condition i) there exists $V \in \mathcal{T}_{\Phi^{*}} \backslash\{\emptyset\}$ such that $V \subset W$ and $V \cap A=\emptyset$. At the same time we get contradiction with the fact that $V \subset \mathrm{cl}_{\mathcal{T}_{\Phi^{*}}}(A)$.

Now, we shall prove that ii) $\Rightarrow$ iii). Let us suppose that $A \notin \mathcal{J}$. Then, by Proposition 1.5, $A \cap \Phi^{*}(A) \in \mathcal{T}_{\Phi^{*}} \backslash\{\emptyset\}$ and we have the contradiction with the fact that $\operatorname{int}_{\mathcal{T}_{\Phi^{*}}}\left(\operatorname{cl}_{\mathcal{T}_{\Phi^{*}}}(A)\right)=\emptyset$.

The implication iii) $\Rightarrow$ i) left to complete the proof. Let $A \in \mathcal{J}$ and $W \in \mathcal{T}_{\Phi^{*}} \backslash\{\emptyset\}$. Remark 2.3 gives that $V=W \backslash A \in \mathcal{T}_{\Phi^{*}}$. Moreover, since $W \in \mathcal{T}_{\Phi^{*}} \backslash\{\emptyset\}$, we obtain, by Remark 2.3, that $W \notin \mathcal{J}$ and, in consequence, that $V \neq \emptyset$. Clearly, $V \cap A=\emptyset$, so the proof is finished.

The above theorem gives that the notions of a nowhere dense set and a strong nowhere dense set in the space $\left\langle X, \mathcal{T}_{\Phi^{*}}\right\rangle$ are equivalent. What is more, we see at once that

Corollary 2.8. If $\mathcal{N}\left(\mathcal{T}_{\Phi^{*}}\right)$ is the family of all nowhere dense sets in $\left\langle X, \mathcal{T}_{\Phi^{*}}\right\rangle$ then $\mathcal{N}\left(\mathcal{T}_{\Phi^{*}}\right)=$ J.

Proposition 2.9. Let $\mathcal{B} a\left(\mathcal{T}_{\Phi^{*}}\right)$ be the smallest $\sigma$-algebra of sets containing the family $\mathcal{N}\left(\mathcal{T}_{\Phi^{*}}\right) \cup \mathcal{T}_{\Phi^{*}}$. Then

$$
\mathcal{B} a\left(\mathcal{T}_{\Phi^{*}}\right)=2^{X} .
$$

Proof. Let $A \subset X$. Then $A=\left(A \backslash \Phi^{*}(A)\right) \cup\left(A \cap \Phi^{*}(A)\right)$. By Proposition 1.3 and Corollary 2.8, $A \backslash \Phi^{*}(A) \in \mathcal{N}\left(\mathcal{T}_{\Phi^{*}}\right)$ and by Proposition $2.5, A \cap \Phi^{*}(A) \in \mathcal{T}_{\Phi^{*}}$, so that $\mathcal{B} a\left(\mathcal{T}_{\Phi^{*}}\right)=2^{X}$.

Proposition 2.10. Let $A \subset X$. Then $A \in \mathcal{J}$ if and only if $A$ is $\mathcal{T}_{\Phi^{*-}}$ closed and $\mathcal{T}_{\Phi^{*-}}$ nowhere dense.

Proof. Let $A \in \mathcal{J}$. Obviously $X \in \mathcal{T}_{\Phi^{*}}$, so, by Remark 2.3, we have that $X \backslash A \in \mathcal{T}_{\Phi^{*}}$.

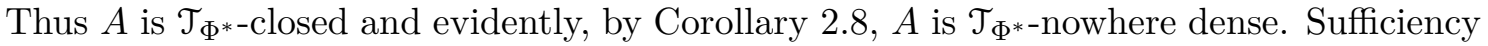
is the consequence of Corollary 2.8.

As the consequence of this property we have

Proposition 2.11. If $A \in \mathcal{J}$ then $A$ is $\mathcal{T}_{\Phi^{*}-\text { closed and }} \mathcal{T}_{\Phi^{*}-\text { discrete. }}$

Also the following property is obvious.

Property 2.12. If $\mathcal{J}$ is a $\sigma$-ideal and $A \in \mathcal{T}_{\Phi^{*}} \backslash\{\emptyset\}$ then $A$ is $\mathcal{T}_{\Phi^{*}-\text { second category, i.e. }}$ $A \notin \mathbb{K}\left(\mathcal{T}_{\Phi^{*}}\right)$.

Moreover, we have

Proposition 2.13. If $\mathcal{J}$ is a $\sigma$-ideal then a set $A \subset X$ is $\mathcal{T}_{\Phi^{*}-\text { compact if and only if } A \text { is }}$ finite.

Proof. Sufficiency is obvious. Let us assume that $A \subset X$ is $\mathcal{T}_{\Phi^{*} \text {-compact }}$ and infinite. Let $B \subset A$ be infinite and countable. Then $(X \backslash B) \cup\{x\} \in \mathcal{T}_{\Phi^{*}}$ for every $x \in B$. Indeed, clearly $B \in \mathcal{J}$ and $\{x\} \in \mathcal{J}$ for any $x \in B$, so $X \triangle((X \backslash B) \cup\{x\}) \in \mathcal{J}$ and, in consequence, Proposition 1.3 Conditions $1^{\circ}$ and $3^{\circ}$ give that $(X \backslash B) \cup\{x\} \subset X=\Phi^{*}((X \backslash B) \cup\{x\})$. Thus $(X \backslash B) \cup\{x\} \in \mathcal{T}_{\Phi^{*}}$. Obviously the family $\{(X \backslash B) \cup\{x\}\}_{x \in B}$ is an open cover of $A$ which does not contain a finite subcover of $A$. It contradicts the fact that $A$ is $\mathcal{T}_{\Phi^{*} \text {-compact. }}$

Proposition 2.14. If $\mathcal{J}$ is a $\sigma$-ideal then the space $\left\langle X, \mathcal{T}_{\Phi^{*}}\right\rangle$ neither fulfills the first nor the second axiom of countability and is not separable.

Proof. Let us suppose that $\left\langle X, \mathcal{T}_{\Phi^{*}}\right\rangle$ fulfills the first axiom of countability. Let $x \in X$

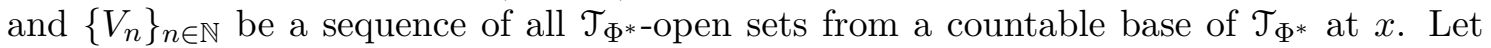
$x_{n} \in V_{n} \backslash\{x\}$ for $n \in \mathbb{N}$. Putting $V=V_{1} \backslash\left\{x_{n}: n \in \mathbb{N}\right\}$ we get that $V \in \mathcal{T}_{\Phi^{*}}, x \in V$ and $V$ 
does not contain any set $V_{n}$ for $n \in \mathbb{N}$. Hence $\left\langle X, \mathcal{T}_{\Phi^{*}}\right\rangle$ does not fulfill the first countability axiom and therefore does not fulfill the second countability axiom. Since every countable set belongs to $\mathcal{J}$ so thus we infer that $\left\langle X, \mathcal{T}_{\Phi^{*}}\right\rangle$ is not separable.

Proposition 2.15. If $\mathcal{J}$ contains an uncountable set then $\left\langle X, \mathcal{T}_{\Phi^{*}}\right\rangle$ is not a Lindelöf space.

Proof. Let $D \in \mathcal{J}$ be an uncountable set then $(X \backslash D) \cup\{x\} \in \mathcal{T}_{\Phi^{*}}$ for every $x \in D$ and $\{(X \backslash D) \cup\{x\}\}_{x \in D} \in \mathcal{T}_{\Phi^{*}}$ is an open cover of $X$ which does not contain a countable subcover.

Since $V=X \backslash\{x\} \in \mathcal{T}_{\Phi^{*}}$ for any $x \in X$, we see at once

Proposition 2.16. The space $\left\langle X, \mathcal{T}_{\Phi^{*}}\right\rangle$ is a $T_{1}$-space.

We end this section with the interesting property of the functions continuous with respect to the generalized topology $\mathcal{T}_{\Phi^{*}}$.

Theorem 2.17. If $\mathcal{J}$ is a $\sigma$-ideal then for an arbitrary function $f: X \rightarrow Y$, where $\langle Y, \mathcal{T}\rangle$ satisfies the second countability axiom, there exists a set $A \in \mathcal{J}$ such that for every $x \in X \backslash A$ the function $f$ is $\mathcal{T}_{\Phi^{*}-\text { continuous at }} x$.

Proof. Let $\left\{B_{n}\right\}_{n \in \mathbb{N}}$ be a countable base of $\langle Y, \mathcal{T}\rangle$. For every $n \in \mathbb{N}$ we have that $f^{-1}\left(B_{n}\right)=C_{n} \cup D_{n}$, where $C_{n}=\Phi^{*}\left(f^{-1}\left(B_{n}\right)\right) \cap f^{-1}\left(B_{n}\right)$ and $D_{n}=f^{-1}\left(B_{n}\right) \backslash \Phi^{*}\left(f^{-1}\left(B_{n}\right)\right)$.

By Proposition 2.5, $C_{n} \in \mathcal{T}_{\Phi^{*}}$ for any $n \in \mathbb{N}$. Moreover, by Proposition 1.3, $A=\bigcup_{n=1}^{\infty} D_{n} \in$ J. Let $x_{0} \in X \backslash A$ and $W \in \mathcal{T}_{\Phi^{*}}$ be such that $f\left(x_{0}\right) \in W$. Thus there exists $n_{0} \in N$ such that $B_{n_{0}} \subset W$ and $x_{0} \in f^{-1}\left(B_{n_{0}}\right)$. Hence $x_{0} \in C_{n_{0}} \in \mathcal{T}_{\Phi^{*}}$ and $f\left(C_{n_{0}}\right) \subset W$. It means that $f$ is $\mathcal{T}_{\Phi^{*} \text {-continuous for every }} x \in X \backslash A$.

\section{The $(\star)$ property and the $(\star \star)$ property}

In this section we concentrate on the family $\mathcal{T}_{\Phi^{*}}$ in a space $\langle X, \mathcal{S}, \mathcal{J}\rangle$ having two special properties: the $(\star)$ property and the $(\star \star)$ property. We start with the definition of these properties.

Definition 3.1. We shall say that $\langle X, \mathcal{S}, \mathcal{J}\rangle$ has

- the $(\star)$ property if there exist $B \subset X$ such that $X \in \mathcal{H}(B) \cap \mathcal{H}(X \backslash B)$;

- the ( $\star \star)$ property if for every $A \subset X$ there exist $B \subset A$ and $C \in \mathcal{H}(B)$ such that $C \in \mathcal{H}(A \backslash B) \cap \mathcal{H}(A)$.

It is easy to see that $\langle X, \mathcal{S}, \mathcal{J}\rangle$ has the $(\star \star)$ property if for every $A \subset X$ there exists $B \subset A$ such that $\mathcal{H}(B) \cap \mathcal{H}(A \backslash B) \cap \mathcal{H}(A) \neq \emptyset$. Moreover, we see at once that if $\langle X, \mathcal{S}, \mathcal{J}\rangle$ has the (*) property and $B \subset X$ is such that $\mathcal{H}(B) \cap \mathcal{H}(X \backslash B) \neq \emptyset$ then $\mathcal{H}(B) \cap \mathcal{H}(X \backslash B)=\{X\}$.

Let $\mathcal{B} a, \mathcal{B}$ and $\mathbb{K}$ be the family of all sets having the Baire property, the family of Borel sets and the family of all meager sets with respect to the natural topology $\tau_{0}$, respectively. Note that the measurable space $\langle\mathbb{R}, \mathcal{B} a, \mathbb{K}\rangle$ has the $(\star)$ property. Indeed, if $\mathfrak{C} \subset \mathbb{R}$ is a Bernstein set then $\mathbb{R} \in \mathcal{H}(\mathfrak{C}) \cap \mathcal{H}(\mathbb{R} \backslash \mathfrak{C})$.

Moreover if additivity of $\sigma$-ideal $\mathbb{K}$ is equal to $\mathfrak{c}$ then the measurable space $\langle\mathbb{R}, \mathcal{B} a, \mathbb{K}\rangle$ has the ( $\star \star)$ property. Indeed, if $A \subset \mathbb{R}$ and $A \in \mathbb{K}$, then for any $B \subset A$ we have that $A \in \mathcal{H}(B) \cap \mathcal{H}(A \backslash B) \cap \mathcal{H}(A)$. If $A \subset \mathbb{R}$ and $A \notin \mathbb{K}$, then the cardinality of the family $\mathfrak{F}=\{F \in \mathcal{B}: A \cap F \notin \mathbb{K}\}$ equals $\mathfrak{c}$. Therefore, one can find sets $P_{1}=\left\{x_{\alpha}: \alpha<\mathfrak{c}\right\}$ and $P_{2}=\left\{y_{\alpha}: \alpha<\mathfrak{c}\right\}$ such that $P_{1} \cup P_{2} \subset A, P_{1} \cap P_{2}=\emptyset$, the cardinality of $P_{1}$ and $P_{2}$ is equal to $\mathfrak{c}$ and $P_{1} \cap F \neq \emptyset \neq P_{2} \cap F$ for any $F \in \mathfrak{F}$. Putting $B=P_{1}$ we obtain that $\mathcal{H}(B) \cap \mathcal{H}(A \backslash B) \cap \mathcal{H}(A) \neq \emptyset$. Indeed, let $V \in \mathcal{H}(A)$. Let $W \subset V \backslash B$ have the Baire property. Suppose that $W \cap A \notin \mathbb{K}$. Obviously, one can find a set $Z \in \mathcal{B}$ such that $Z \subset W$ and $Z \cap A \notin \mathbb{K}$. Thus $\emptyset \neq Z \cap P_{1} \subset W \cap P_{1}$, which is impossible. Therefore, we have that $W \cap A \in \mathbb{K}$ and, in consequence, $W \backslash A$ has the Baire property. Since $V \in \mathcal{H}(A)$, we 
obtain that $W \backslash A \in \mathbb{K}$. Hence $W \in \mathbb{K}$. Finally, we have that $V \in \mathcal{H}(B)$. By a similar argument, $V \in \mathcal{H}(A \backslash B)$.

Theorem 3.2. If $\langle X, \mathcal{S}, \mathcal{J}\rangle$ has the $(\star)$ property then the smallest topology $\sigma\left(\mathcal{T}_{\Phi^{*}}\right)$ containing $\mathcal{T}_{\Phi^{*}}$ is equal to $2^{X}$.

Proof. Let $x \in X$ and $B \subset X$ be such that $\mathcal{H}(B) \cap \mathcal{H}(X \backslash B) \neq \emptyset$. Thus $B \cup\{x\} \in \mathcal{T}_{\Phi^{*}}$ and $(X \backslash B) \cup\{x\} \in \mathcal{T}_{\Phi^{*}}$. It implies that $(B \cup\{x\}) \cap((X \backslash B) \cup\{x\}) \in \sigma\left(\mathcal{T}_{\Phi^{*}}\right)$, so that $\sigma\left(\mathcal{T}_{\Phi^{*}}\right)=2^{X}$.

Theorem 3.3. If $\langle X, \mathcal{S}, \mathcal{J}\rangle$ has the $(\star)$ property then $\mathcal{T}_{\Phi^{*}}$ does not include the supremum of the topologies included in $\mathcal{T}_{\Phi^{*}}$.

Proof. Let us suppose that $\mathcal{T}$ is the supremum of the topologies included in $\mathcal{T}_{\Phi^{*}}$. Let $B \subset X$ be such that $X \in \mathcal{H}(B) \cap \mathcal{H}(X \backslash B)$. Let $x_{0} \in X$. Put $\mathcal{T}_{1}=\left\{\emptyset, B \cup\left\{x_{0}\right\}, X\right\}$ and $\mathcal{T}_{2}=\left\{\emptyset,(X \backslash B) \cup\left\{x_{0}\right\}, X\right\}$. It is easy to see that $\mathcal{T}_{1}, \mathcal{T}_{2}$ are topologies contained in $\mathcal{T}_{\Phi^{*}}$, so $\mathcal{T}_{1} \cup \mathcal{T}_{2} \subset \mathcal{T}$. Moreover, $\left(B \cup\left\{x_{0}\right\}\right) \cap\left((X \backslash B) \cup\left\{x_{0}\right\}\right)=\left\{x_{0}\right\} \in \mathcal{T} \subset \mathcal{T}_{\Phi^{*}}$. It is a contradiction with the fact that $\left\{x_{0}\right\} \notin \mathcal{T}_{\Phi^{*}}$.

However, we have the following property.

Theorem 3.4. There exists a maximal topology in the family $\mathcal{A}$ of all topologies contained in $\mathcal{T}_{\Phi^{*}}$ and ordered by the inclusion.

Proof. Let $\left\{\mathcal{T}_{\lambda}\right\}_{\lambda \in \Lambda}$ be an arbitrary chain in $\mathcal{A}$. Put $\mathcal{T}=\left\{\bigcup_{w \in W} A_{w}:\left\{A_{w}\right\}_{w \in W} \subset \bigcup_{\lambda \in \Lambda} \mathcal{T}_{\lambda}\right\}$. We see at once that $\emptyset, X \in \mathcal{T}, \mathcal{T}$ is closed under arbitrary unions and $\mathcal{T} \subset \mathcal{T}_{\Phi^{*}}$. Since $\left\{\mathcal{T}_{\lambda}\right\}_{\lambda \in \Lambda}$ is a chain we obtain that $\mathcal{T}$ is closed under finite intersections. Therefore $\mathcal{T}$ is a topology contained in $\mathcal{T}_{\Phi^{*}}$ and simultaneously, it is the upper bound of $\left\{\mathcal{T}_{\lambda}\right\}_{\lambda \in \Lambda}$. By Kuratowski-Zorn Lemma we get the existence of a maximal topology in $\mathcal{A}$.

Proposition 3.5. If $\langle X, \mathcal{S}, \mathcal{J}\rangle$ has the $(\star)$ property then $\left\langle X, \mathcal{T}_{\Phi^{*}}\right\rangle$ is a Hausdorff space.

Proof. Let $x, y \in X$ and $x \neq y$. Let $B \subset X$ be such that $X \in \mathcal{H}(B) \cap \mathcal{H}(X \backslash B)$. If $x \in B$ and $y \in X \backslash B$ then putting $V_{1}=B$ and $V_{2}=X \backslash B$ we get that $V_{1}, V_{2} \in \mathcal{T}_{\Phi^{*}}, V_{1} \cap V_{2}=\emptyset$, $x \in V_{1}$ and $y \in V_{2}$. If $x, y \in B$ then it is enough to consider the sets $V_{1}=B \backslash\{y\} \in \mathcal{T}_{\Phi^{*}}$ and $V_{2}=(X \backslash B) \cup\{y\} \in \mathcal{T}_{\Phi^{*}}$. If $x, y \in X \backslash B$ the proof runs in the similar way.

Proposition 3.6. If $\langle X, \mathcal{S}, \mathcal{J}\rangle$ has the $(\star \star)$ property then $\left\langle X, \mathcal{T}_{\Phi^{*}}\right\rangle$ is a normal space.

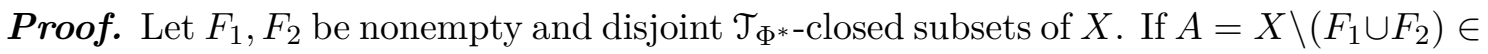
$\mathcal{J}$ then putting $V_{1}=\left(X \backslash F_{2}\right) \backslash A$ and $V_{2}=\left(X \backslash F_{1}\right) \backslash A$ we get that $F_{1} \subset V_{1}, F_{2} \subset V_{2}$, $V_{1}, V_{2} \in \mathcal{T}_{\Phi^{*}}$ and $V_{1} \cap V_{2}=\emptyset$.

If $A \notin \mathcal{J}$ then by the $(\star \star)$ property there exist $B \subset A$ and $C \in \mathcal{H}(B)$ such that $C \in \mathcal{H}(A \backslash B) \cap \mathcal{H}(A)$. Let $V_{1}=F_{1} \cup B, V_{2}=F_{2} \cup(A \backslash B)$. Evidently, $F_{1} \subset V_{1}$, $F_{2} \subset V_{2}$ and $V_{1} \cap V_{2}=\emptyset$. We prove first that $V_{1} \in \mathcal{T}_{\Phi^{*}}$. In this purpose we show that $F_{1} \cup B \subset \Phi^{*}\left(F_{1} \cup B\right)$. Since $\Phi^{*}\left(F_{1} \cup B\right)=\Phi^{*}\left(F_{1} \cup C\right)=\Phi^{*}\left(F_{1} \cup A\right)=\Phi^{*}\left(X \backslash F_{2}\right)$. Suppose that $x \in\left(F_{1} \cup B\right) \backslash \Phi^{*}\left(F_{1} \cup B\right) \subset X \backslash \Phi^{*}\left(X \backslash F_{2}\right)$. Because $F_{2}$ is $\mathcal{T}_{\Phi^{*} \text {-closed it means }}$ that $X \backslash F_{2} \subset \Phi^{*}\left(X \backslash F_{2}\right)$ and finally $x \in\left(F_{1} \cup B\right) \backslash\left(X \backslash F_{2}\right)=\left(F_{1} \cup B\right) \backslash\left(F_{1} \cup A\right)=\emptyset$. This contradiction infer that $V_{1} \in \mathcal{T}_{\Phi^{*}}$. Similarly, we can prove that $V_{2} \in \mathcal{T}_{\Phi^{*}}$. It ends the proof.

Theorem 3.7. If $\langle X, \mathcal{S}, \mathcal{J}\rangle$ has the $(\star \star)$ property then every $\mathcal{T}_{\Phi^{*}-\text { closed subset of } X \text { is }}$ $G_{\delta^{-}}$set in the space $\left\langle X, \mathcal{T}_{\Phi^{*}}\right\rangle$.

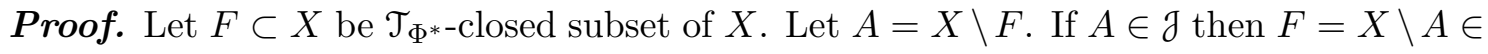
$\mathcal{T}_{\Phi^{*}}$

Let us assume that $A \notin \mathcal{J}$. By the ( $\star \star)$ property there exist $B \subset A$ and $C \in \mathcal{H}(B)$ such that $C \in \mathcal{H}(A \backslash B) \cap \mathcal{H}(A)$. Let $V_{1}=F \cup B$ and $V_{2}=F \cup(A \backslash B)$. Simultaneously as in the proof of the previous theorem we get that $V_{1}, V_{2} \in \mathcal{T}_{\Phi^{*}}$. Since $F=V_{1} \cap V_{2}$, we get that $F$ is $G_{\delta^{-}}$set in the space $\left\langle X, \mathcal{T}_{\Phi^{*}}\right\rangle$. 


\section{References}

[1] Á. Császár, Generalized open sets, Acta Math. Hungar. 75, 65-87, 1997.

[2] Á. Császár, Generalized topology, generalized continuity, Acta Math. Hungar. 96 (4), 351-357, 2002.

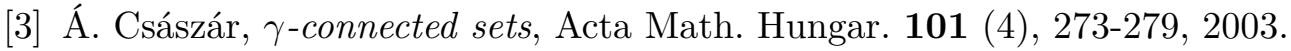

[4] Á. Császár, Separation axioms for generalized topologies, Acta Math. Hungar. 104 (1-2), 63-69, 2004.

[5] O. Haupt and C. Pauc, La topologie de Denjoy envisagée comme vraie topologie, C. R. Acad. Sci. Paris 234, 390-392, 1952.

[6] J. Hejduk, S. Lindner and A. Loranty, On lower density type operators and topologies generated by them, Filomat 32 (14), 4949-4957, 2018.

[7] J. Hejduk and R. Wiertelak, On the abstract of density topologies generated by lower and almost lower density operators, Traditional and present-day topics in real analysis, University of Łódź, 431-440, 2013.

[8] T. Jyothis and J.J. Sunil, $\mu$-Compactness in Generalized Topological Spaces, J. Adv. Stud. Topol. 3 (3), 18-22, 2012.

[9] E. Korczak-Kubiak, A. Loranty and R.J. Pawlak, Baire generalized topological spaces, generalized metric spaces and infinite games, Acta Math. Hungar. 140 (3), 203-231, 2013.

[10] S. Lindner and M. Terepeta, On the Position of Abstract Density Topologies in the Lattice of All Topologies, Filomat 30 (2), 281-286, 2016.

[11] J. Lukeš, J. Malý and L. Zajíček, Fine topology methods, Real Analysis and Potential Theory, Lecture Notes in Math. 1189, Springer-Verlag, Berlin, 1986.

[12] J. Oxtoby, Measure and Category, Springer-Verlag, New York, 1987.

[13] W. Wilczyński, Density topologies, Handbook of Measure Theory, Ed. E. Pap. Elsevier, chapter 15, 675-702, 2002. 